\title{
Preliminary results of leaf litter-decomposing microfungi survey
}

\author{
Barry Katz \\ University of North Carolina
}

As part of an OEA-sponsored collaboration with $\mathrm{Dr}$. Rolf Singer, I have been collecting microfungi from decomposing Dicotyledonous leaf litter. Eight collections were made from four Terra Firme communities, tree collections from an Igapó, and two each from Campinarana and Varzea communities. In addition, there was a collection of permamently submerged litter from Tarumãzinho. Each collection contained approximately 300 fungal isolates. In this preliminary survey 4,500 fungi were isolated.

Among the dozen taxa recovered at all sites there were members of the cosmopolitan genera Acremonium, Aspergillus, Chloridium, Paecilomyces, Penicillium, Pestalotia, Scolecobasidium, and Trichoderma. Other ubiquitous taxa include Beltrania rhombica, Thozetella spp, unidentified dematiaceous Hyphomycete D 6436 and unidentified Ascomycete 7512 .

Besides the above-mentioned taxa, only seven taxa were isolated from two or more plant community types. These include the unusual dematiaceous Hyphomycetes Chaetopsina fulva, Gliocephalotrichum bulbiferum and Phaeoisaria clematididis. Preliminary examination of the other taxa failed to produce appropriate generic dispositions .

There were more than one hundred taxa of sporulating fungi which were isolated from individual plots. These include species of Charala, Cladosporium, Codinaea, Dactylaria, Exophiala, Fusarium, Geotrichum, Gliocladium, Monodictys, Phialocephala, Phialophora, Rhinocladiella, Scolecobasidium, Septonema, Sporothrix \& Stachybotrys. The distribution of Basidiomycetous hyphae isolated with clamp connections was also restricted to individual collecting sites.

While 300 isolates per collection appears to be inadequate to monitor total deversity there is a great deal of localization of fungi. Sporulating taxa from duplicate collections of a plot were as different from each other as collections from different community types. While future collections will inevitably show that many more fungi are widely distributed, other localized "endemics" are certain to be isolated as well. These preliminary results should alert the reader of the mycological lite- 
rature to view general statements about Tropical litter-decomposing fungi with caution. Future work will focus on the distribution patterns of microfungi in Terra Firme and Igapó communities. Leaf colonization in the canopy will be examined, also.

\section{REsumo}

Nas observaçốes iniciais dos microfungos decompositores de folhas de dicotiledôneas da liteira, coletados na terra firme, igapó, campinarana e várzea, alèm da liteira submersa do Tarumãzinho, foram isolados 4.500 fungos. Representantes dos gẻneros cosmopolitas Acremonium, Aspergillus, Chloridium, Paecilomyces, Penicilvium, Pestalotia, Scolecobasidium e Trichoderma, assim como Beltrania rhombica, Thozetella spp., Hyphomycetes D 6436 e Ascomycetes 7512 foram encontrados em todos os ambientes. Em dois ou mais ambientes foram encontrados os não comuns Chaetopsina fulva, Gliocephalotrichum bulbiferum e Phaeoisaria clematididis. Entre os mais de cem isolados de uma única amostra, es. tâo incluidas espécies de Charala, Cladosporium, Codinaea, Dactylaria , Exophiala, Fusarium, Geotrichum. Gliocladium. Monodictys, Phialocephala, Phialophora, Rhinocladiella, Scolecobasidium, Septonema, Sporothrix e Stachybotrys. Hifas de Basidiomycetes com fibulas também foram encontradas em um único local. Os 300 fungos isolados por coleção parecem nảo ser suficientes para demonstrar a diversidade dos mesmos. Taxa de coleçōes duplicadas de um mesmo local eram tảo diferentes quanto os de coleçōes de tipos vegetacionais diferentes. Futuras coleçōes demonstrarāo inevitavelmente que muitos fungos sāo largamente distribuidos e outros "endêmicos". Estes resultados preliminares deverăo servir como alerta aos especialistas em Micologia a terem cuidado quanto aos seus pontos de vista com relaçăo aos fungos tropicais decompositores da liteira.

(Aceito para publicaçāo em 17/09/80) 